\title{
Extending the robustness and efficiency of artificial compressibility for partitioned fluid-structure interactions
}

\author{
AEJ Bogaers ${ }^{1,2}, \mathrm{~S} \mathrm{Kok}^{3}$, BD Reddy ${ }^{4,5}, \mathrm{~T}_{\text {Franz }}^{4,6}$ \\ ${ }^{1}$ Advanced Mathematical Modelling, Modelling and Digital Sciences, CSIR, South Africa \\ ${ }^{2}$ Department of Mechanical Engineering, University of Cape Town, South Africa \\ ${ }^{3}$ Department of Mechanical and Aeronautical Engineering, University of Pretoria, South Africa \\ ${ }^{4}$ Centre for Research in Computational and Applied Mechanics, University of Cape Town, South Africa \\ ${ }^{5}$ Department of Mathematics and Applied Mathematics, University of Cape Town, South Africa \\ ${ }^{6}$ Cardiovascular Research Unit, Chris Barnard Division of Cardiothoracic Surgery, University of Cape Town, \\ South Africa
}

\begin{abstract}
In this paper we introduce the idea of combining artificial compressibility (AC) with quasi-Newton (QN) methods to solve strongly coupled, fully/quasi-enclosed fluid-structure interaction (FSI) problems. Partitioned, incompressible, FSI based on Dirichlet-Neumann domain decomposition solution schemes cannot be applied to problems where the fluid domain is fully enclosed. A simple example often provided in literature is that of a balloon with a prescribed inflow velocity. In this context, artificial compressibility (AC) is a useful method by which the incompressibility constraint can be relaxed by including a source term within the fluid continuity equation. The attractiveness of $\mathrm{AC}$ stems from the fact that this source term can readily be added to almost any fluid field solver, including most commercial solvers. Once included, both the modified fluid solver and structural solver can be treated as "black-box" field operators. AC is however limited to the class of problems it can effectively be applied to. For example, AC is an efficient solution strategy for the simulation of blood flow through arteries, but performs poorly when applied to the simulation of blood flow through an opening heart valve. The focus of this paper is thus to extend the application of AC by including an additional Newton system accounting for the missing interface sensitivities. We do so through the use of a multi-vector update quasi-Newton (MVQN) method, where the required system Jacobians are approximated rather than explicitly computed. In so doing, we continue to facilitate the notion that the AC modified fluid field solver and solid field solver can be treated as "black-box" solvers. We aim to demonstrate the improved performance of the combination of $\mathrm{AC}+\mathrm{QN}$ when compared to $\mathrm{AC}$ applied in isolation.
\end{abstract}

\section{keywords}

Fluid-structure interactions, partitioned solver, artificial compressibility, quasi-Newton methods, implicit coupling 


\section{Introduction}

Fluid Structure Interaction (FSI) remains an active research field in engineering and life science. Specifically, incompressible-flow FSI problems are the focus of many publications. For these types of problems, partitionedsolution schemes remain popular as they allow for the re-use of existing fluid and solid solvers. Coupling schemes that allow for the use of "black-box" solvers further increase the range of valid solver choices. While these schemes aren't always competitive in comparison with monolithic solvers [11], several coupling schemes have been proposed that can efficiently solve strongly-coupled-incompressible FSI problems $[4,12,14,15,17,19,20,22,26]$.

The majority of partitioned schemes are based on Dirichlet-Neumann (DN) domain decomposition. DN schemes are classified by the fluid domain interface inheriting a displacement/velocity interface condition where interface tractions (Neumann conditions) are imposed along the solid domain interface. One particular limitation of DN based algorithms arise when considering fully enclosed, incompressible fluid domains, coined by Küttler et al. [21] as the "incompressibility dilemma". An incompressible fluid domain with a prescribed inlet flow and fully enclosed by a Dirichlet type interface manifests itself as an ill-posed problem. Common examples of the incompressibility dilemma include balloon inflation type problems. Numerical difficulties however are also present when considering quasi-enclosed problems, for example flow through a collapsible tube or the simulation of opening and closing heart valves.

Several potential solutions to the incompressibility dilemma have been proposed. Küttler et al. [21] suggest reformulating the solid equations as a volume constraint problem. Robin-Robin (or Robin-Neumann) boundary conditions have been mentioned (though not as of yet demonstrated) as a plausible solution [3]. Degroote et al. [13], and Råback et al. [23] have proposed modifying the continuity equation to include an artificial compressibility term. While these methods are certainly reasonable solutions, they require modifications to the source code. To the best of the authors' knowledge, reversing the direction of information transfer (i.e. Neumann-Dirichlet boundary conditions) remains as the only viable means by which black-box partitioned solutions schemes can allow for fully enclosed incompressible FSI problems. However, for stiff structures, the sensitivity of interface forces w.r.t. displacements (and vice versa) become too large for standard black-box coupling schemes [21].

Artificial compressibility (AC) is however an interesting option in the context of black-box solvers. The source code modifications for the implementation of $\mathrm{AC}$ is minimal, and requires the addition of a scalar source term to the continuity equation. Most commercial solvers, including for example Fluent [1], allow for the addition of source terms via user defined functions, negating the need to have direct access to the source code. Similarly, if the source code were available, the AC term can often be included without the need for the user becoming involved with the internal solver specific solution procedures. AC as a method therefore still resides within the "spirit" of black-box coupling, where almost all stand alone fluid solvers remain available for use.

When considered as a method in the context of FSI, artificial compressibility (AC/FSI) does have some limitations. The additional source term can be based on a linearized approximation of the solid domain's volume change in response to a change in pressure $[10,13,18]$. AC/FSI is therefore well suited to problems where this relationship bears relevance to the nature of the FSI problem, see for example [18], where AC/FSI was applied to solve for blood flow through a bifurcating arterial section. There are however many problems where this relationship is entirely unsuitable. One such example, as mentioned in [10], is the simulation of flow around a flexible beam. A small localized pressure perturbation at the beam tip will result in the entire length of the beam deforming (as opposed to a small localized tip displacement as would be suggested by the linearized pressure-volume approximation). Furthermore, while the proposed pressure-volume approximation (which we will describe in greater detail in the sections to follow) often results in the near optimal computation of an appropriate coefficient choice, it is based on a linearized approximation. As such, AC as a method, is often incapable of accurately accounting for many of the non-linearities present within an FSI system, especially when there are large inertial forces present in the structural domain sub-problem.

In this paper we propose the possibility of combining AC with quasi-Newton (QN) methods. The AC/FSI modification would allow for problems with fully/quasi-enclosed domains to be solved. The additional quasi-Newton sensitivities would then further stabilize the method. It does so by continuing to treat the solid domain solver as a complete black-box, requiring only interface displacement information as a function of imposed interface tractions. Similarly, it treats the AC modified fluid equations as a black-box.

We will specifically focus on the implementation of a new multi-vector update quasi-Newton method (MVQN) 
introduced in [6]. The MVQN method was specifically designed for transient strongly coupled partitioned FSI problems (though the method is likely applicable to a much broader class of problems). While the primary purpose of the paper is the introduction of AC/FSI + QN methods, it serves the additional purpose of proof of robustness with regards to the newly proposed MVQN approximation method.

We will demonstrate the performance of the proposed coupling scheme via numerical experiments, for both fully enclosed balloon inflation type problems and a well known benchmark problem of flow induced oscillations of a flexible beam. We will demonstrate that the proposed combination of AC/FSI + QN methods not only improves on the performance of $\mathrm{AC}$ but can at times yield near-quadratic convergence rates. The paper will initially introduce $\mathrm{AC} / \mathrm{FSI}$, including the interface test load method to approximate the AC coefficients. The new MVQN Jacobian updating method will then be outlined as well as the application of QN methods to AC/FSI. The paper will finally conclude with performance evaluations via numerical examples.

\section{Coupled Partitioned FSI Problem}

FSI is described by a set of highly non-linear coupled equations involving both a fluid and solid domain, $\Omega^{F}$ and $\Omega^{S}$ respectively. The two domains share a common, non overlapping interface $\Gamma_{\mathrm{FSI}}$. In an abstract formulation, each of the respective field solvers may be viewed as an interface operator. The solid solver is therefore an interface operator $\boldsymbol{S}$ mapping a given interface force vector $\boldsymbol{f}_{\Gamma}^{F, n+1}$ to interface displacements

$$
\boldsymbol{d}_{\Gamma}^{S, n+1}=\boldsymbol{S}\left(\boldsymbol{f}_{\Gamma}^{F, n+1}\right)
$$

where $\boldsymbol{d}_{\Gamma}^{S, n+1}$ is the interface displacement vector at time step $n+1$. Similarly, the fluid field solver is represented by a mapping operator $\boldsymbol{F}$ such that

$$
\boldsymbol{f}_{\Gamma}^{F, n+1}=\boldsymbol{F}_{\mathrm{AC}}\left(\boldsymbol{d}_{\Gamma}^{S, n+1}\right) .
$$

The fluid field operator $\boldsymbol{F}$ denotes both the solution step of the fluid field variables as well as the mesh movement of the fluid domain nodal coordinates. The subscript AC is included here to indicate that the fluid operator includes the additional artificial compressibility source term. In this paper we limit our discussion to partitioned FSI based on fluid equations that are described within an Arbitrary Lagrangian-Eulerian (ALE) framework, described in greater detail in the section to follow.

For the FSI problem, it is essential that both the kinematic and dynamic continuity be satisfied at all times along the interface. Satisfying kinematic continuity ensures mass conservation where dynamic continuity leads to the conservation of linear momentum. In order to guarantee energy conservation, in a partitioned setting, it therefore becomes important to iterate on the two domain solutions (1) and (2) to within a specified convergence tolerance. In the case of no-slip boundary conditions on the moving interface, kinematic continuity states that the fluid flow velocity at the interface $\boldsymbol{u}_{\Gamma}$ equals the boundary displacement/velocity

$$
\boldsymbol{u}_{\Gamma}=\frac{\partial \boldsymbol{d}_{\Gamma}}{\partial t}
$$

and dynamic continuity states that the interface stress states $\sigma$ are equal at the interface,

$$
\boldsymbol{\sigma}_{\Gamma}^{S} \cdot \boldsymbol{n}=\boldsymbol{\sigma}_{\Gamma}^{F} \cdot \boldsymbol{n}
$$

where $\boldsymbol{n}$ is the respective interface normals and the superscripts indicate the solid $(S)$ or fluid $(F)$ domains.

\subsection{Artificial Compressibility (AC)}

Artificial compressibility in the context of fluid structure interactions (AC/FSI) was originally introduced by Riemslagh et al. [24] in a 1D FSI framework as a means by which to stabilize the coupling between incompressible fluids and elastic walls. The method has since been applied to more complex problems $[13,18,23]$. AC/FSI can be viewed as a variation of the original $\mathrm{AC}$ algorithm first proposed in [7] as a means to couple the momentum and continuity 
equations and in turn stabilize incompressible Navier-Stokes equations. With regards to AC/FSI, a pressure time derivative is inserted into the continuity equation, where this additional term is based on a physical approximation of the pressure-volume response of the structure. Not only does this additional term have a stabilizing effect on the non-linear FSI equations, but it also serves as a means by which to resolve the incompressibility dilemma.

In order to introduce the AC modifications, let us first define our fluid equations which form the basis of our fluid interface operator $\boldsymbol{F}$. The incompressible Navier-Stokes equations, in an Arbitrary Lagrangian-Eulerian (ALE) framework that accounts for the moving fluid domain, is given by

$$
\begin{gathered}
\frac{\partial \boldsymbol{u}}{\partial t}+\left(\boldsymbol{u}-\boldsymbol{u}_{m}\right) \cdot \nabla \boldsymbol{u}-\nu \Delta \boldsymbol{u}+\nabla p=\boldsymbol{f} \\
\nabla \cdot \boldsymbol{u}=\mathbf{0} .
\end{gathered}
$$

Here $\boldsymbol{u}$ is the fluid velocity, $\boldsymbol{u}_{m}$ the ALE-coordinate system velocity at a given reference position, $p$ the kinematic pressure, $\nu$ the kinematic viscosity and $f$ accounts for the body forces.

The basic idea of artificial compressibility applied to FSI, is to modify the continuity equation (6) by inserting a pressure time derivative such that

$$
\beta\left(\frac{\partial p}{\partial t}\right)+\nabla \cdot \boldsymbol{u}=\mathbf{0}
$$

where $\beta$ is the artificial compressibility coefficient. Following the work of Råback et al. [23], (7) is equivalent to allowing the fluid density to vary with pressure such that $d \rho / \rho=\beta d p$. Inserting the pressure density relation into the compressible flow continuity equation $\partial \rho / \partial t+\nabla \cdot \boldsymbol{u}=0$, and neglecting the spatial derivative of $\rho$ yields a continuity equation of the form given in (7).

Equation (7) does not require additional pseudo-iterations to solve as suggested by [21]. Instead the solution can be viewed as an iteration trick, where the time derivative of pressure is approximated in a given iteration via

$$
\frac{\beta\left(p_{k}^{n+1}-p_{k-1}^{n+1}\right)}{\Delta t}+\nabla \cdot \boldsymbol{u}_{k}^{n+1}=\mathbf{0}
$$

where subscript $k$ represents the current FSI coupling iteration in time step $n+1$ and $\Delta t$ is the simulation time step size. Therefore, while the incompressible continuity equation (6) is initially violated, at convergence for time step $n+1$, the AC compressibility term disappears as $p_{k}^{n+1} \rightarrow p_{k-1}^{n+1}$, thereby satisfying the original continuity equation.

The AC/FSI method relies heavily on an optimal choice of $\beta$. Assuming the structure is defined by a linear set of equations, it would be possible to define a linear relationship between the fluid domain volume change $\Delta V$ related to a corresponding interface pressure change $\Delta p$ such that

$$
\frac{\Delta V}{V}=\beta \Delta p .
$$

Using the relation (9), it is then possible to construct an analytical expression for $\beta$ (see for example [23] for flow through a flexible tube). Analytical expressions are however limited to simple geometries. To allow for the AC/FSI method to be generally applicable to more complex geometries, both Järvinen et al. [18] and Degroote et al. [13] have suggested using an interface test load method.

Given two different pressures $p_{1}$ and $p_{0}$ applied along the wetted interface $\Gamma_{\mathrm{FSI}}$, two distinct volumes $V_{1}$ and $V_{0}$ would be obtained. $\beta$ may then be approximated by a finite difference approximation

$$
\beta=\frac{1}{V_{0}} \frac{\left(V_{1}-V_{0}\right)}{\left(p_{1}-p_{0}\right)} .
$$

While the approximation (10) suggests application of AC throughout the entire enclosed domain, AC can equally be applied along the interface only. Interface-artificial-compressibility (IAC), following the work of Degroote et al. [13] limits AC to the elements adjacent to the interface. While AC throughout the domain significantly simplifies the numerical properties of the Navier-Stokes equations, IAC has been demonstrated to produce slightly superior FSI convergence behavior [5]. In fact, it is possible to draw some parallel similarities between IAC/FSI and Robin 
boundary conditions, where the fluid equations along the interface are augmented with an approximation of the solid domain sensitivities [10]. For the purposes of this paper we will exclusively use IAC.

The IAC approximation of $\boldsymbol{\beta}$ requires the projected volume change of the interface elements. For small deformations, a numerically convenient way in which to approximate $\boldsymbol{\beta}$ based on this projected volume change is

$$
\boldsymbol{\beta}=\frac{\Delta \boldsymbol{L}}{\boldsymbol{h}} \frac{1}{\Delta p}
$$

where $\Delta \boldsymbol{L}=\Delta \boldsymbol{d}_{\Gamma} \cdot \boldsymbol{n}_{\Gamma}$, the perpendicular change in interface displacement. $\boldsymbol{h}$ is the equivalent fluid element height which can be computed as any given element's interface volume to area ratio in 3D (or area to length ratio in $2 \mathrm{D}$ )

$$
h_{i}=\frac{V_{i}}{A_{\Gamma, i}}, \text { for } i=1,2, \ldots, N_{\Gamma} .
$$

It is possible that $\boldsymbol{\beta}$ may be computed as a constant (or averaged value). We however treat $\boldsymbol{\beta}$ as a non-uniform field allowing for better approximations for solid domains that have either varying structural properties or geometrically varying volume-pressure relationships.

It should be noted that the choices for $p_{1}$ and $p_{0}$ have little effect on the computed approximation of $\boldsymbol{\beta}$. In order to allow for a fair comparison of $\mathrm{AC}$, the coefficient $\boldsymbol{\beta}$ is recomputed at the start of each time step. If the normal interface displacements are appropriately transfered to the fluid interface, then (11) can be computed using two structural solver calls only, as opposed to two full FSI iterations. Furthermore, while not demonstrated in the results to follow, when AC/FSI is combined with QN methods, there is very little difference in performance of $\mathrm{AC} / \mathrm{FSI}+\mathrm{QN}$ if $\boldsymbol{\beta}$ is adaptively updated each time step or computed once-off at the start of the simulation (and remains static for the duration of the simulation).

As a final note, shear stresses are ignored within the IAC approximation, but not in the actual simulation. This can be done without overly affecting the stabilizing effect of AC because pressure forces along the interface, for most FSI problems, dominate (shear forces typically account in the order of 1 percent of the total interface forces). $\mathrm{AC} / \mathrm{FSI}$ is therefore unsuited to problems that are shear driven.

\subsection{IAC/FSI + quasi-Newton}

IAC is a useful way in which the fluid equations can be modified to allow for the solution of fully/quasi-enclosed problems. The method is however largely only applicable to problems where the pressure-volume relationship bears relevance to the nature of the coupled problem. Furthermore, while the test load method provides a means by which to compute the optimal (or near optimal) value for the AC coefficient, AC/FSI includes only a linearized approximation of the structural system behavior. Interrogating equations (7) and (11) it may be noted that AC/FSI and the test load method does not for example account for the structural inertial terms. Therefore, the ability to compute what is demonstrably the near optimal AC coefficient, does not guarantee that AC/FSI will not produce sub-optimal convergence behavior for certain problems.

In this section we propose augmenting the IAC/FSI method through the use of quasi-Newton (QN) methods. The IAC terms included into the fluid equations are an approximation of the solid domain's sensitivities w.r.t. fluid interface pressures. IAC in isolation however ignores the additional fluid sensitivities w.r.t. the domain displacements. The aim therefore of the additional QN terms is to include these sensitivities. We will show that not only will this reduce the sensitivity to the computed values of $\boldsymbol{\beta}$, but will also provide improved convergence behavior of IAC/FSI, thus potentially allowing IAC to be applied to a much larger range of problems.

In order to describe the QN method, let use first rewrite our fluid system mapping operator (2) as a root finding problem, such that

$$
\boldsymbol{r}_{F}=\boldsymbol{F}_{A C}(\boldsymbol{d}(\boldsymbol{f}))-\boldsymbol{f}=\mathbf{0},
$$

where the functional dependency of displacements on forces is now indicated by $\boldsymbol{d}(\boldsymbol{f})$. We can now define a Newton system such that 


$$
\left[\begin{array}{ll}
\frac{\partial \boldsymbol{F}}{\partial \boldsymbol{d}} \frac{\partial \boldsymbol{d}}{\partial \boldsymbol{f}}-\boldsymbol{I} & \frac{\partial \boldsymbol{F}}{\partial \boldsymbol{d}}
\end{array}\right]\left\{\begin{array}{c}
\Delta \boldsymbol{f} \\
\Delta \boldsymbol{d}
\end{array}\right\}=[-\boldsymbol{F}(\boldsymbol{d}(\boldsymbol{f}))+\boldsymbol{f}]
$$

which re-arranged results in a block-Newton equation to solve for the fluid force update

$$
\left[\frac{\partial \boldsymbol{F}}{\partial \boldsymbol{d}} \frac{\partial \boldsymbol{d}}{\partial \boldsymbol{f}}-\boldsymbol{I}\right] \Delta \boldsymbol{f}=-(\boldsymbol{F}(\boldsymbol{d}(\boldsymbol{f}))-\boldsymbol{f})-\frac{\partial \boldsymbol{F}}{\partial \boldsymbol{d}} \Delta \boldsymbol{d}
$$

An update for the interface tractions is then found by

$$
\boldsymbol{f}_{k+1}^{n+1}=\boldsymbol{f}_{k}^{n+1}+\Delta \boldsymbol{f}
$$

The updated interface tractions $\boldsymbol{f}_{k+1}^{n+1}$ is then transferred to the solid interface solver, which is now based on the additional sensitivities of the fluid solver.

It is important to note here, because of the QN update step, that the starting pressure for the modified AC equations be updated as well. Recall from equation (8) that the modified fluid continuity equation is now

$$
\frac{\boldsymbol{\beta}\left(\boldsymbol{p}_{k}^{n+1}-\boldsymbol{p}_{k-1}^{n+1}\right)}{\Delta t}+\nabla \cdot \boldsymbol{u}_{k}^{n+1}=\mathbf{0} .
$$

At the start of the next FSI coupling iterate in time step $n+1, \boldsymbol{p}_{k-1}^{n+1}$ will have been modified because of the QN update (15). $\boldsymbol{p}_{k-1}^{n+1}$ should therefore also be modified based on the pressure component of $\Delta \boldsymbol{f}$ such that

$$
\boldsymbol{p}_{k-1, \text { new }}^{n+1}=\boldsymbol{p}_{k-1}^{n+1}+\Delta \boldsymbol{f} \cdot \boldsymbol{n}_{\Gamma} .
$$

Without this pressure correction a Newton-like method in conjunction with AC/FSI cannot work.

In order to solve the block-Newton system described by equation (14), we now wish to construct approximations of the fluid and solid system Jacobians $\boldsymbol{J}_{F}=\frac{\partial \boldsymbol{F}}{\partial \boldsymbol{d}}=\frac{\partial \boldsymbol{f}}{\partial \boldsymbol{d}}$ and $\boldsymbol{J}_{S}=\frac{\partial \boldsymbol{S}}{\partial \boldsymbol{f}}=\frac{\partial \boldsymbol{d}}{\partial \boldsymbol{f}}$. In this paper we will make use of the multi-vector update quasi-Newton (MVQN) method introduced in [6]. The MVQN method has demonstrated itself to be both robust and efficient for a large class of partitioned transient FSI problems.

In order to facilitate the discussion, let us assume that we have completed $k$ FSI coupling iterations within time step $n+1$. We have therefore performed $k$ fluid solver calls, where $k$ interface displacements were transferred to the fluid solver which subsequently provided $k$ corresponding interface tractions. Using the displacement and traction observations, we can now construct two differencing observations matrices

$$
\begin{gathered}
\Delta \boldsymbol{D}_{F}^{n+1}=\left[\boldsymbol{d}_{F, k-1}-\boldsymbol{d}_{F, k}, \boldsymbol{d}_{F, k-2}-\boldsymbol{d}_{F, k}, \ldots, \boldsymbol{d}_{F, 1}-\boldsymbol{d}_{F, k}\right] \\
\Delta \boldsymbol{F}_{F}^{n+1}=\left[\boldsymbol{f}_{F, k-1}-\boldsymbol{f}_{F, k}, \boldsymbol{f}_{F, k-2}-\boldsymbol{f}_{F, k}, \ldots, \boldsymbol{f}_{F, 1}-\boldsymbol{f}_{F, k}\right],
\end{gathered}
$$

where the subscript $F$ denotes information with regards to the fluid field solver. To simplify notation, the superscript $n+1$ was dropped from each of the respective observation vectors.

The MVQN method is based on an iterative updating scheme, whereby all the information/observations from the current time step is exactly matched, while information from previous time steps is matched in a minimum norm sense. Starting with an initial Jacobian from the previous time step $\boldsymbol{J}^{n}$, we iteratively update the Jacobian based on an update rule of the form

$$
\boldsymbol{J}_{F, k+1}^{n+1}=\tilde{\boldsymbol{J}}_{F, k+1}^{n+1}+\boldsymbol{J}_{F}^{n},
$$

where $\tilde{\boldsymbol{J}}_{F, k+1}^{n+1}$ denotes the Jacobian update approximation. We can then construct a secant equation for $\tilde{\boldsymbol{J}}_{F, k+1}^{n+1}$

$$
\tilde{\boldsymbol{J}}_{F, k+1}^{n+1} \Delta \boldsymbol{F}_{F}^{n+1}=\Delta \boldsymbol{D}_{F}^{n+1}-\boldsymbol{J}_{F}^{n} \Delta \boldsymbol{F}_{F}^{n+1},
$$

where solving for the minimum of $\left\|\tilde{\boldsymbol{J}}_{F, k+1}^{n+1}\right\|$ subject to the constraint of (21), we obtain the Jacobian update rule 


$$
\boldsymbol{J}_{F, k+1}^{n+1}=\boldsymbol{J}_{F}^{n}+\left(\Delta \boldsymbol{D}_{F}^{n+1}-\boldsymbol{J}_{F}^{n} \Delta \boldsymbol{F}_{F}^{n+1}\right)
$$

$$
\left(\left(\Delta \boldsymbol{F}_{F}^{n+1}\right)^{T} \Delta \boldsymbol{F}_{F}^{n+1}\right)^{-1}\left(\Delta \boldsymbol{F}_{F}^{n+1}\right)^{T}
$$

$\|\boldsymbol{J}\|$ refers to the norm on the vector space of matrix $\boldsymbol{J}$. It is important to note that the matrix inversion ()$^{-1}$ in equation (22) is of size $[k \times k]$ (the number of coupling iterations within the given time step). While the matrix system is sufficiently small to warrant being solved using matrix inversion, it is advised that the linear system $\left(\left(\Delta \boldsymbol{F}_{F}^{n+1}\right)^{T} \Delta \boldsymbol{F}_{F}^{n+1}\right)^{-1}\left(\Delta \boldsymbol{F}_{F}^{n+1}\right)^{T}$ rather be solved via factorization, to limit the effects of ill-conditioning should any be present.)

The displacement vectors in (18) would typically be the displacements received as output from the solid field solver transferred to the fluid interface. However, because of artificial compressibility, there is in fact now an additional "perceived" displacement field in addition to the true boundary displacement. This additional displacement field can be included to the fluid displacement vectors such that

$$
\boldsymbol{d}_{F, k}=\boldsymbol{d}_{F, k}+\boldsymbol{u}_{A C}
$$

where $\boldsymbol{u}_{A C}$ incorporates the normal displacement accounted for by AC. The $\boldsymbol{u}_{A C}$ term may be approximated by

$$
\boldsymbol{u}_{A C, k, i}^{n+1}=\left(\beta_{i}\left(p_{F, k, i}^{n+1}-p_{F, k-1, i}^{n+1}\right) h_{i}\right) \cdot \boldsymbol{n}_{\Gamma, k, i}
$$

$$
\text { for } i=1,2, \ldots, N_{\Gamma} \text {, }
$$

where $N_{\Gamma}$ is the number of elements along the interface. The additional $\boldsymbol{u}_{A C}$ includes the $\boldsymbol{\beta}$ dependency into the fluid system quasi-Newton approximation. The term in essence accounts for the fact that $\boldsymbol{F}_{A C}$ is not only dependent on displacement, $\boldsymbol{F}(\boldsymbol{d})$, but is also functionally dependent on the modified starting pressure, i.e. $\boldsymbol{F}_{A C}\left(\boldsymbol{d}_{S, k}, \boldsymbol{p}_{F, k-1}\right)$. However, since $\boldsymbol{J}_{F}$ is already only based on an approximation, $\boldsymbol{u}_{A C}$ can be safely ignored and will only minimally affect the overall convergence behavior. In the numerical examples we will illustrate the effect of including the additional AC displacement field, referred to as option A, where option B will refer to the omission of the $\boldsymbol{u}_{A C}$ term.

In a similar fashion, $\boldsymbol{J}_{S}$ is approximated by constructing two observation matrices of the interface tractions and displacements related to the solid field solver

$$
\begin{array}{ll}
\Delta \boldsymbol{F}_{S}^{n+1}= & {\left[\boldsymbol{f}_{S, k}-\boldsymbol{f}_{S, k+1}, \boldsymbol{f}_{S, k-2}-\boldsymbol{f}_{S, k+1}, \ldots, \boldsymbol{f}_{S, 1}-\boldsymbol{f}_{S, k+1}\right]} \\
\Delta \boldsymbol{D}_{S}^{n+1}=\quad & \\
& {\left[\boldsymbol{d}_{S, k}-\boldsymbol{d}_{S, k+1}, \boldsymbol{d}_{S, k-2}-\boldsymbol{d}_{S, k+1}, \ldots, \boldsymbol{d}_{S, 1}-\boldsymbol{d}_{S, k+1}\right] .}
\end{array}
$$

The solid Jacobian $\boldsymbol{J}_{S}$ is then similarly approximated via the update

$$
\boldsymbol{J}_{S, k+1}^{n+1}=\boldsymbol{J}_{S}^{n}+\left(\Delta \boldsymbol{F}_{S}^{n+1}-\boldsymbol{J}_{S}^{n} \Delta \boldsymbol{D}_{S}^{n+1}\right)
$$

$$
\left(\left(\Delta \boldsymbol{D}_{S}^{n+1}\right)^{T} \Delta \boldsymbol{D}_{S}^{n+1}\right)^{-1}\left(\Delta \boldsymbol{D}_{S}^{n+1}\right)^{T} .
$$

Because the MVQN method requires that at least two coupling iterations have been performed in order to construct a Jacobian approximation, for the first iteration of the first time step a fixed point iteration with only 
AC present is employed. The Jacobian update rules (22) and (27) further requires the availability of $\boldsymbol{J}^{n}$. Since we do not have an available starting Jacobian, we set $\boldsymbol{J}_{F}^{0}=[\mathbf{0}]$ and $\boldsymbol{J}_{S}^{0}=[\mathbf{0}]$ for the first iteration in time step 1. For the first iteration of subsequent time steps the approximate Jacobian from the preceding time step is used but the update formulae are only applied from iteration count 2 onwards. A summary of the IAC/FSI + MVQN algorithm is provided as pseudo code in Appendix A.

The MVQN updating method is ideally suited to approximate Jacobians for transient problems. In the absence of exact information, any closely related information is often beneficial. The MVQN method allows for the approximations to be constructed using all the information from all the completed time steps (including all iterations within each of these time steps). If any information from the current time step falls along the same direction as information from a preceding time step, the older information is naturally replaced. This in turn removes the possibility of constructing approximations based on contradictory information. Furthermore, unlike other similar quasi-Newton methods, the MVQN method will always be able to exactly match all observations from the current time step. Information from previous time steps are matched in a minimum-norm sense only and is as a consequence naturally less emphasized. The method therefore has a certain inherent consistency with Newton's method w.r.t. to the current time step (the point about which the equations are linearized). For a comparison of the MVQN method to more established quasi-Newton methods including Broyden's method and the IBQN-LS reduced order modeling method applied to partitioned FSI problems see [6].

As a side note, several publications pertaining to DN partitioned schemes base the system Jacobians on the displacement residual equation (see for example [20]). A similar notion here would be to solve for the residual equations of the fluid field forces such that

$$
\left[\frac{\partial \boldsymbol{r}}{\partial \boldsymbol{f}}\right]_{k} \Delta \boldsymbol{f}=-\boldsymbol{r}_{k}
$$

where $\boldsymbol{r}_{k}=\boldsymbol{f}_{k}-\boldsymbol{f}_{k-1}$, and $\left[\frac{\partial \boldsymbol{r}}{\partial \boldsymbol{f}}\right]_{k}$ is the Jacobian for the current coupling iteration. The Jacobian for the residual equations can then be approximated using the MVQN method or other similar QN methods. The linearized system described by (28) can be advantageous from a numerical efficiency perspective. The inverse of the system Jacobian can be directly approximated thereby removing the need for a linear system solve. From our experience however, this is not a feasible alternative when considering the modified $\mathrm{AC}$ fluid equations. Because of the $\mathrm{AC}$ terms, the fluid interface forces are now directly linked to displacement. The relation between two successive interface tractions (on the basis of directions and magnitudes alone) are therefore insufficient to describe the system behavior.

To better explain this, let us consider the fluid equations without AC. Given a particular displacement $\boldsymbol{d}_{\Gamma, k}^{n+1}$, with the exclusion of the AC term, will always produce the same $\boldsymbol{f}_{\Gamma, k}^{n+1}$. On the other hand, with the inclusion of the $\mathrm{AC}$ term, a given displacement $\boldsymbol{d}_{\Gamma, k}^{n+1}$ may now yield different interface forces depending on the interface force guess from the previous iteration. Hence the necessity to include the system sensitivities of forces w.r.t. displacements and displacements w.r.t. to forces, as described by the block-Newton equation (14).

\section{Test Problems}

In this section we analyze artificial compressibility as well as the combination with quasi-Newton methods, via numerical examples. OpenFOAM [2], an open source finite volume solver, is used for the fluid domain solution, while Calculix [16], an open source finite element method (FEM) solver, was used for the solid domain. The primary focus of the paper is on a comparison and analysis of the computational efficiencies and convergence behavior of the various coupling strategies. Issues surrounding solution accuracies and temporal convergence receives only rudimentary attention as both AC/FSI and QN methods have independently been verified in several publications. The fluid solver, including the additional AC terms, is based on a modified pimpleDyMFoam solver (available in OpenFOAM). The time integration schemes employed is a first order backward-Eular scheme for the fluid domain, and a generalized alpha scheme for the solid domain. It is therefore to be expected that the overall temporal convergence behavior of the FSI solution scheme be limited by the fluid solver.

Both mesh movement and interface information transfer is performed using radial basis function interpolation. Mesh movement is performed such that the entire fluid domain is included thereby removing the necessity for 


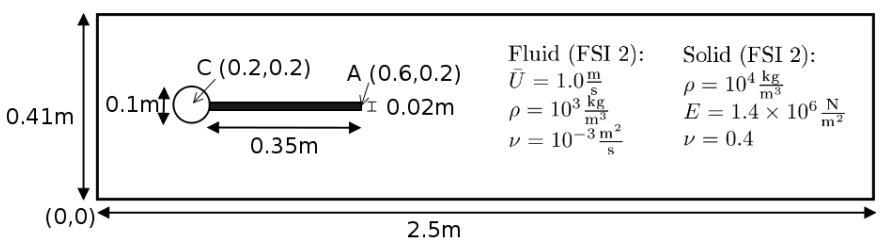

Figure 1: 2D Flexible beam problem description .

remeshing [8]. Interface information transfer is performed using a consistent formulation, thus allowing for a constant stress state to be exactly transferred (i.e. satisfy a patch test) [9].

The convergence criterion employed for the test problems is of the form

$$
\frac{\left\|\boldsymbol{d}_{\Gamma, k+1}-\boldsymbol{d}_{\Gamma, k}\right\|}{\sqrt{m}} \leq \epsilon
$$

where $m$ is the number of DOFs along the interface and is included to remove the dependency of the solution residual to the interface mesh size, and $\epsilon$ is a suitably chosen constant.

\subsection{D Flexible Beam}

The first problem we analyze is flow around a fixed cylinder, with an attached flexible beam. The problem was first proposed by Turek et al. [25] with fairly substantial numerical verifications. The flexible beam problem, as already mentioned in [10], represents an example of a class of problems for which AC/FSI is not well suited to solve. We therefore hope to demonstrate that the inclusion of the additional QN terms are sufficiently accurate to provide efficient coupling behavior.

The problem layout and material descriptions is provided in Figure 1. In this paper we only analyze the transient FSI problem referred to as FSI2 in [25]. FSI2 consists of a $0.02 \mathrm{~m}$ thick, $0.35 \mathrm{~m}$ long flexible beam attached to a fixed circular cylinder with a $0.1 \mathrm{~m}$ diameter. The fluid domain has a parabolic inflow with mean velocity of $\bar{U}=1.0 \frac{\mathrm{m}}{\mathrm{s}}$. The properties of the problem is such that the flexible beam oscillates as a result of vortex development following the fixed cylinder. The center of the cylinder is by design constructed to be non-symmetric to remove dependence on numerical errors for the onset of any oscillations. The inlet flow velocity for the transient FSI2 is slowly ramped up for $t<0.5 \mathrm{~s}$ via $(1-\cos (\pi t / 2)) / 2$. The top, bottom, fixed cylinder and flexible beam walls are defined as non-slip boundaries, where a reference pressure of 0 is prescribed at the domain outlet.

The fluid domain is discretized using 1725 linear elements, where the solid domain is defined with a geometrically non-linear, plane strain formulation with 72 full-integration, quadratic elements. We make use of the comparatively coarse discretization to allow for the use of comparatively large time step sizes. The problem is solved here for four different time step sizes of $\Delta t=0.01 \mathrm{~s}, \Delta t=0.005 \mathrm{~s}, \Delta t=0.0025 \mathrm{~s}$ and $\Delta t=0.001 \mathrm{~s}$. A convergence criterion of $\epsilon=10^{-8}$ is used across all time step sizes. A plot of the beam tip displacement (point A) is shown in Figure 2, along with a snapshot of the domain deformation and pressure contours at time step 12.58s.

In Table 1 we compare the number of coupling iterations required by each of the coupling schemes as well as a summary of the beam tip displacement for all three time step sizes. Because IAC/FSI is so poorly suited to solve this class of problems, and the problem is not fully enclosed thus enabling standard coupling techniques to be used, we additionally compare the coupling performance of using quasi-Newton methods only. For a more detailed description of the MVQN and it's application to partitioned FSI see [6].

From the results, it is immediately evident that IAC/FSI in isolation is severely limited. For the two coarse time step sizes, the scheme fails to converge entirely. For $\Delta t=0.0025 \mathrm{~s}$, the relative change in displacement between two time steps is sufficiently small to allow for IAC/FSI to provide convergent results, albeit with a very large number of required iterations. The performance of the IAC/FSI+MVQN coupling scheme compares very well with the coupling behavior of using only MVQN. Therefore, despite the fact that the AC terms included within the continuity equation, and the additional test load approximation is in no way suited to solve for a flexible beam type problem, the combination of techniques does allow for a comparatively efficient coupling methodology. 


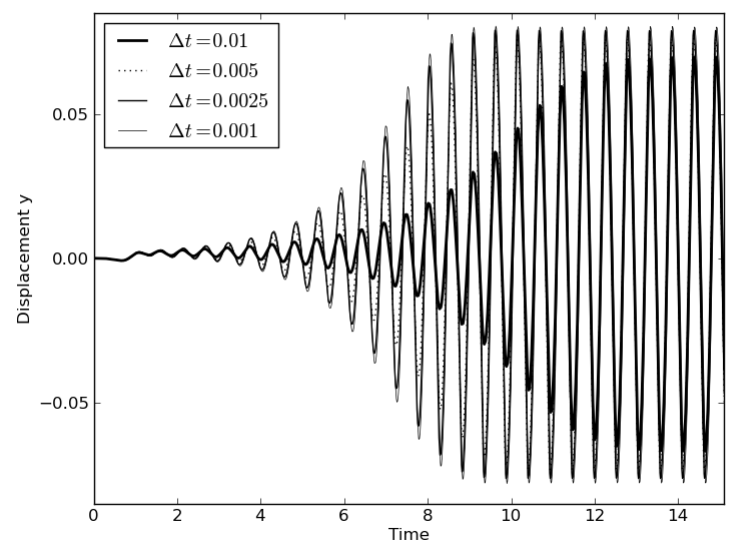

(a)

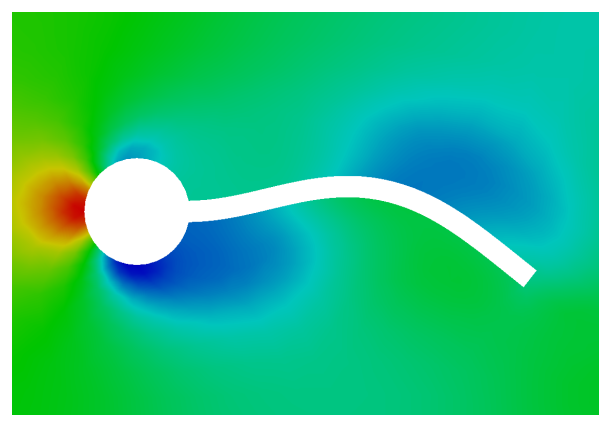

(b)

Figure 2: (a) Beam tip displacement of point A for the flexible beam problem shown for different time steps, along with (b) a snapshot of the beam deformation with pressure contours shown here for time step $12.58 \mathrm{~s}$.

Table 1: Comparison of the minimum, maximum and mean number of iterations required for a convergence tolerance of $\epsilon=10^{-8}$ for the flexible beam benchmark problem. (-) indicates non-convergence and the beam tip displacement at point $\mathrm{A}\left(u_{y}\right)$ is provided in the form of meantamplitude[frequency] and compared to the reference results of Turek et al. [25].

\begin{tabular}{|c|c|c|c|c|c|}
\hline & \# Iterations & MVQN Only & IAC/FSI & IAC/FSI + MVQN & \begin{tabular}{l|l|}
$u_{y}$ & $10^{-3}$ \\
\end{tabular} \\
\hline \multirow{3}{*}{$\Delta t=0.01 \mathrm{~s}$} & Min & 3 & - & 3 & \multirow{3}{*}{$1.44 \pm 68.3[1.9]$} \\
\hline & $\operatorname{Max}$ & 5 & - & 5 & \\
\hline & Mean & 3.28 & - & 3.63 & \\
\hline \multirow{3}{*}{$\Delta t=0.005 \mathrm{~s}$} & Min & 3 & - & 3 & \multirow{3}{*}{$1.40 \pm 74.9[1.9]$} \\
\hline & Max & 5 & - & 5 & \\
\hline & Mean & 3.18 & - & 3.46 & \\
\hline \multirow{3}{*}{$\Delta t=0.0025 \mathrm{~s}$} & Min & 3 & 3 & 3 & \multirow{3}{*}{$1.31 \pm 77.8[1.9]$} \\
\hline & Max & 5 & 129 & 5 & \\
\hline & Mean & 3.27 & 50.58 & 3.48 & \\
\hline \multirow{4}{*}{$\Delta t=0.001 \mathrm{~s}$} & Min & 3 & 3 & 3 & \multirow{3}{*}{$1.20 \pm 79.2[1.9]$} \\
\hline & $\operatorname{Max}$ & 5 & 14 & 6 & \\
\hline & Mean & 3.32 & 6.98 & 3.60 & \\
\hline & & & & Reference [25] & $1.23 \pm 80.6[2.0]$ \\
\hline
\end{tabular}




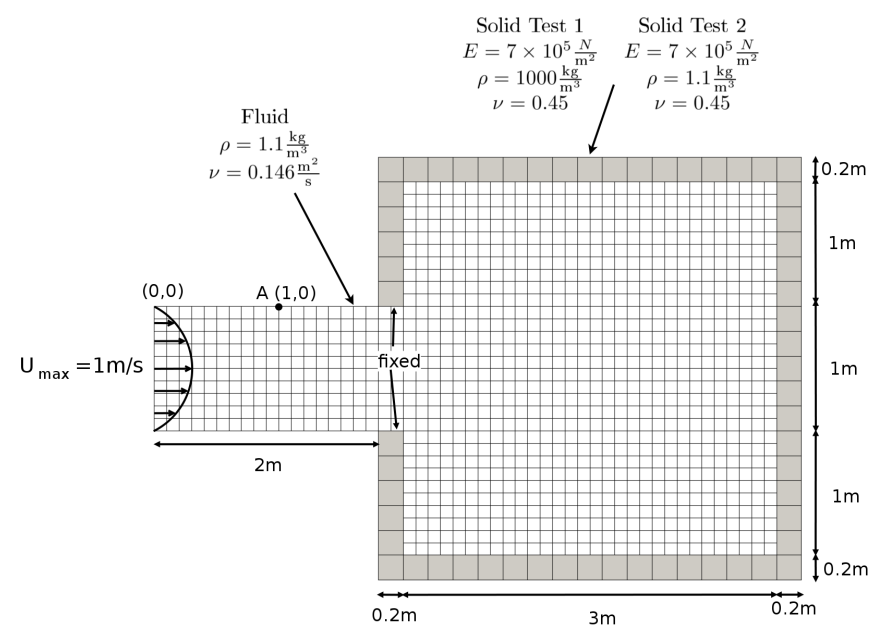

Figure 3: Fully enclosed balloon inflation test problem setup.

\subsection{Balloon Inflation}

We analyze here a balloon inflation test problem first introduced by Küttler et al. [21]. The problem consists of a fully enclosed fluid domain with an inlet flow velocity. The aim of the test problem is to firstly outline the feasibility, associated performance and the limitations of the IAC method. We then demonstrate, by comparison, the potential merits of IAC in conjunction with quasi-Newton methods.

The inflation test problem is outlined in Figure 3 . The problem consists of a $3 \mathrm{~m} \times 3 \mathrm{~m}$ internal volume, enclosed by a $0.2 \mathrm{~m}$ thick shell. A parabolic inflow velocity is applied at the inlet, which is slowly ramped for $t<1 \mathrm{~s}$ from $U_{\max \text { in }}=0$ to $1 \mathrm{~m} / \mathrm{s}$ via the sinusoidal curve $(\sin (\pi(t+3 / 2))+1) / 2$ to avoid spurious pressure oscillations which may occur given a large initial inlet flux. The fluid density and kinematic viscosity used for the numerical experiment is $\rho_{f}=1.1 \mathrm{~kg} / \mathrm{m}^{3}$ and $\nu=0.146 \mathrm{~m}^{2} / \mathrm{s}$. The structural domain is described by a geometrically non-linear formulation and is solved using 59 quadratic full integration elements, where the fluid domain is solved using 1100 linear elements. The simulation is solved for a time step size of $\Delta t=0.05 \mathrm{~s}$.

In order to demonstrate the various associated properties of IAC, we use two different choices of material properties. The first test concerns itself with a comparatively high elastic modulus with a high solid to fluid density ratio. For the second test, likened to the complexity of biomechanics problems, the solid density is reduced to the same as the fluid density. The two sets of solid material properties are provided in Figure 3.

A snapshot of the velocity for Test 1 is shown in Figure 4, along with a plot of pressure at point A. It should be noted, that while reducing the density in Test 2 complicates the problem from a coupling perspective, the pressure at point A remains identical to that of Test 1. The internal pressure state is a function of the structural stress state, which for the balloon inflation problem is a function of the elastic properties. The boundary flux error made in each of the time steps is shown in Figure 5 (for a convergence tolerance of $\epsilon<10^{-7}$ ). The relative boundary flux error can be related directly to the volume error made in each time step, as the flux along the enclosed interface (defined by the deformation of the solid interface), should equal the total flux entering the domain (based here on a prescribed inlet velocity). It is not possible to formulate an analytical solution to the problem, primarily due to the transient nature and the higher order wall motions that occur as a result of the rapidly increasing internal pressure. The relative flux error in each time step does however confirm that continuity remains satisfied (within the limit of convergence tolerance). The maximum flux error is $1.83 \times 10^{-7}$ with a cumulative error of $2.73 \times 10^{-6}$ (which can of course be further improved should the convergence tolerance, time step or discretization be refined).

Performance plots of the investigated methods are shown in Figure 6 along with a summary of both the number of iterations and relative CPU time shown in Table 2. The IAC/FSI method allows for the solution of a fully enclosed, incompressible FSI problem. The performance is however approximately improved by a factor 2 through the inclusion of the additional QN terms. For test 2, the unit fluid-solid density ratio, along with the stiff structure results in a problem which initially provides serious difficulty for IAC in isolation. This can primarily be attributed 


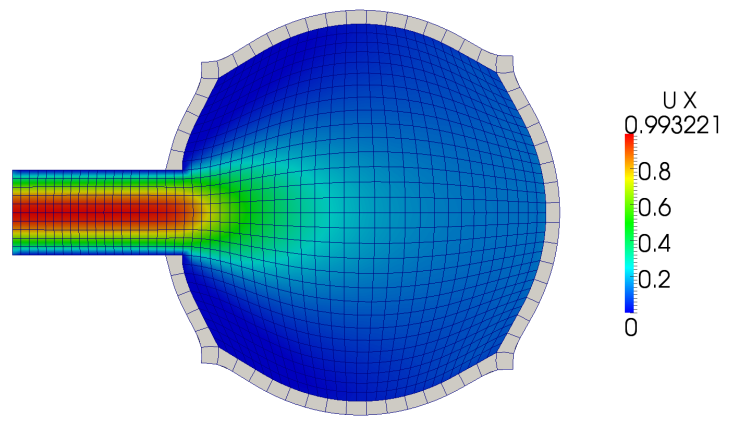

(a)

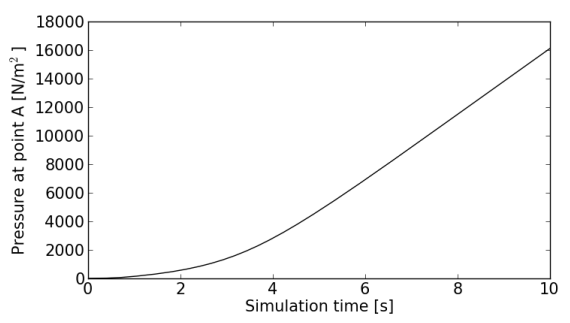

(b)

Figure 4: Balloon inflation results for solid test 1 showing (a) $\boldsymbol{u}_{x}$ contour plot at 10 seconds and (b) pressure level at point $\mathrm{A}$.

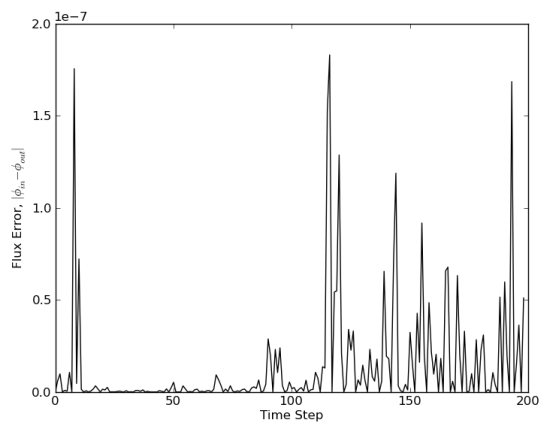

Figure 5: Boundary flux error made in each of the time steps for the balloon inflation test problem. The cumulative error across the entire simulation is $2.73 \times 10^{-6}$ with a maximum error of $1.83 \times 10^{-7}$. 
Table 2: Comparison of the number of iterations and relative computational time for the balloon inflation test problem.

\begin{tabular}{|c|cccc|cccc|}
\cline { 2 - 9 } \multicolumn{1}{c|}{} & \multicolumn{4}{c|}{ Test 1} & \multicolumn{4}{c|}{ Test 2} \\
\hline IAC/FSI & Mean & Min & Max & CPU & Mean & Min & Max & CPU \\
\hline IAC/FSI+MVQN: Option A & 3.94 & 3 & 9 & 2.07 & 10.65 & 6 & 21 & 2.45 \\
IAC/FSI+MVQN: Option B & 4.53 & 3 & 5 & 1.00 & 5.07 & 4 & 8 & 1.04 \\
\hline
\end{tabular}

to the material properties resulting in a coupled problem with very high interface gradients, and an inability of IAC/FSI to properly account for all the non-linearities present within the coupled FSI problem. The addition of the extra QN sensitivities are however sufficiently accurate to overcome this limitation.

It is important to understand that the convergence rates shown in Figure 6 are only representative of the typical convergence behavior for each of the methods. At time step 150, option A (IAC/FSI+MVQN) is capable of producing near quadratic convergence. However, this will not be the case for all time steps. It does nevertheless provide an indication of how good the MVQN Jacobian approximations can be. Additionally, the comparitive results between Test 1 and Test 2 illustrate that the additional $\boldsymbol{u}_{A C}$ term has little influence on the coupling behavior of IAC/FSI+MVQN. For Test 1 we observe a minor benefit if it is included but not so for Test 2 .

Lastly, in Figure 7 we perform an exhaustive search over 3 different time steps illustrating the effect $\boldsymbol{\beta}$ has on the performance of IAC/FSI. The intent of the search is to illustrate that there is a very narrow bandwidth for acceptable AC coefficients. In all the times analyzed here, the test load method does provide the optimal/near optimal solution to $\boldsymbol{\beta}$. Despite this, AC/FSI in isolation does suffer from sub-optimal convergence rates. The inclusion of the additional QN terms not only removes this narrow-bandwidth w.r.t. the computed values of $\boldsymbol{\beta}$, but also reduces the overall number of coupling iterations.

\subsection{Damped Structural Instability Fully Enclosed Test Problem}

The last problem we analyze is a more interesting fully enclosed domain problem than the one analyzed in Section 3.2. The problem's geometry is based on the test problem introduced in [21] and is depicted here in Figure 8. The problem consists of a curved fluid domain which is in turn fully enclosed by thin structural membranes of differing stiffnesses. The fluid domain is loaded with a gravitational body force of $g_{y}=-1 \frac{\mathrm{m}}{\mathrm{s}^{2}}$ and asymmetrical parabolic inflows is prescribed, with the right inflow being slightly higher. The velocities are slowly ramped up for $t<1$.0s via the sinusoidal curve, $(\sin (\pi(t+3 / 2))+1) / 2$. The respective short edges of the structural domains are fixed. The structure is described by a geometrically non-linear FEM formulation and is modeled with 100 quadratic, full integration elements where the fluid domain is modeled using 3600 linear FV elements. The time steps utilized for the simulation is $\Delta t=0.005 \mathrm{~s}$ with convergence criterion of $\epsilon=1 \times 10^{-7}$.

The primary idea behind the problem is that as fluid is introduced into the domain, the top (less stiff) structure initially deforms to accommodate the additional fluid flow. As some critical pressure is reached, the bottom structure eventually collapses. Just like in [21] we solve the problem ignoring the potential cavitation effects which may arise in a problem of this nature, and are again primarily interested in the comparative convergence behavior of the coupling procedures analyzed here.

In Figure 9 we show the velocities along with the domain deformations at various time steps. The corresponding reference pressure at point $\mathrm{A}$ is shown in Figure 10. The results, despite the minor differences in problem setup, correspond qualitatively well to those presented in [21]. As the fluid domain reaches some critical pressure, the bottom structure slowly collapses; with the collapse occurring on the side with a higher inflow velocity. The collapse leads to an initial decrease in internal pressure followed by a period of highly oscillating pressure as the structure and fully enclosed domain undergo rapid deformations. Again, to demonstrate that continuity is satisfied at convergence within each time step we illustrate the flux error made in each time step in Figure 11.

The damped structural instability problem represents an interesting challenge for the artificial compressibility method. Firstly, we have varying structural stiffnesses which highlight the need for a non-constant AC coefficient along the interface. At the same time, the problem, as with the balloon inflation problem, poses a difficulty to the 

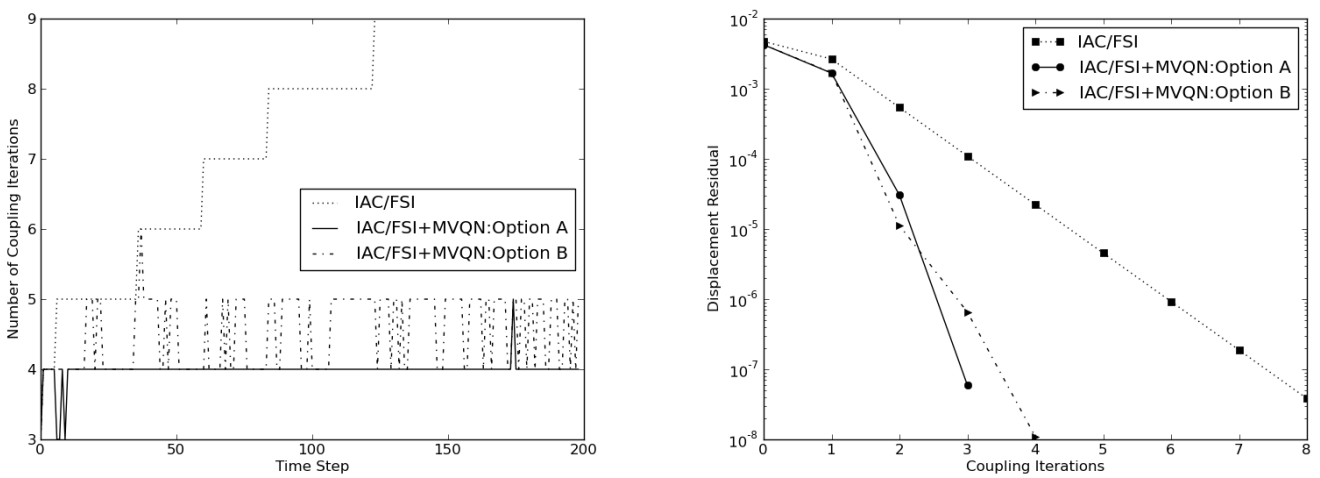

(a)
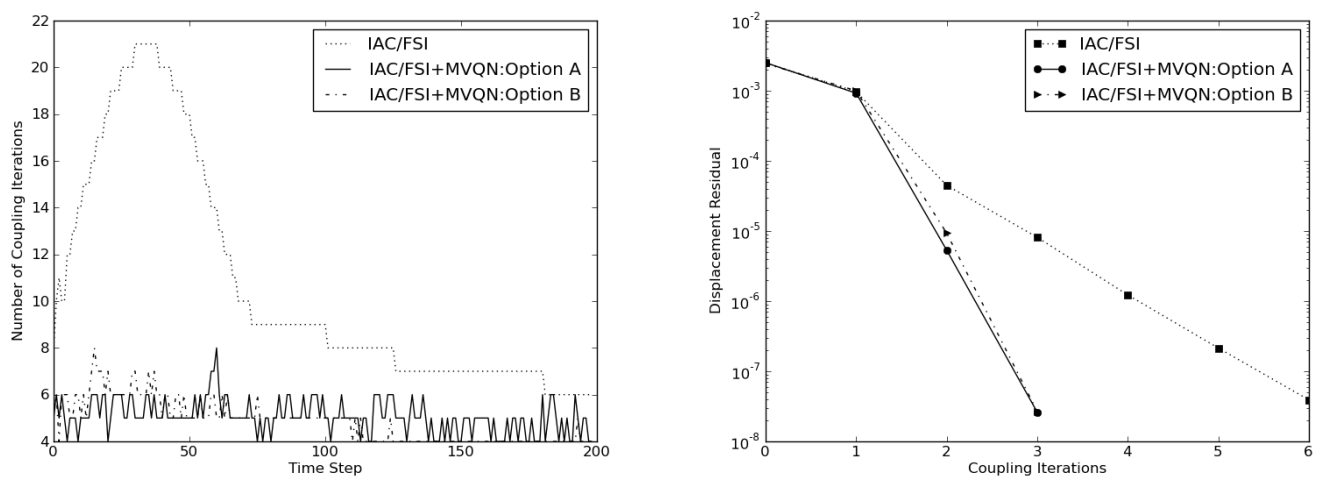

(b)

Figure 6: Performance plots illustrating the number of coupling iterations required for each of the time steps and the typical convergence behavior for each of the methods (shown for time step 150) for the balloon inflation test problem for (a) Test $1\left(E=7 \times 10^{5} \mathrm{kN} / \mathrm{m}^{2}, \rho_{s}=1000 \mathrm{~kg} / \mathrm{m}^{3}, \rho_{f}=1.1 \mathrm{~kg} / \mathrm{m}^{3}\right)$ and (b) Test $2\left(E=7 \times 10^{5} \mathrm{kN} / \mathrm{m}^{2}, \rho_{s}=\right.$ $\left.1.1 \mathrm{~kg} / \mathrm{m}^{3}, \rho_{f}=1.1 \mathrm{~kg} / \mathrm{m}^{3}\right)$. 


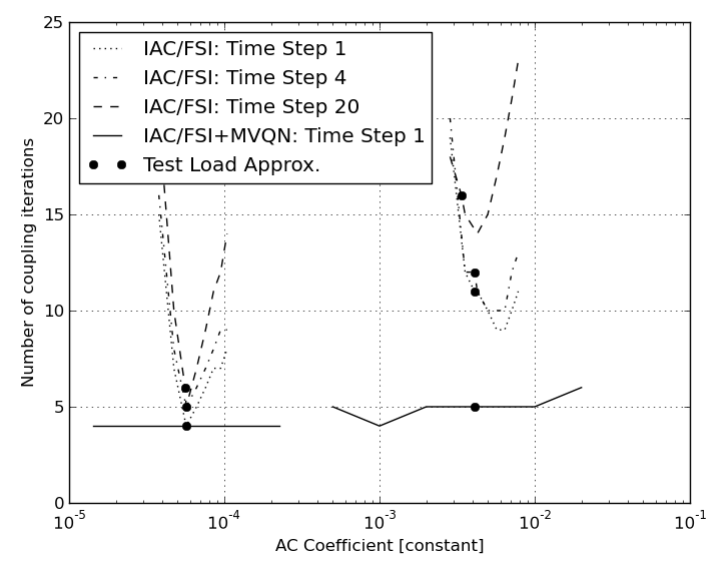

Figure 7: Effect of $\boldsymbol{\beta}$ on the number of coupling iterations, shown for three different time steps. On the left are the results for test 1 with test 2 results on the right. $\boldsymbol{\beta}$ for the exhaustive search is computed as a constant field value. Notice the fine bandwidth of acceptable values of $\boldsymbol{\beta}$ for the IAC/FSI implementation and the smoothing effect of the additional QN terms.

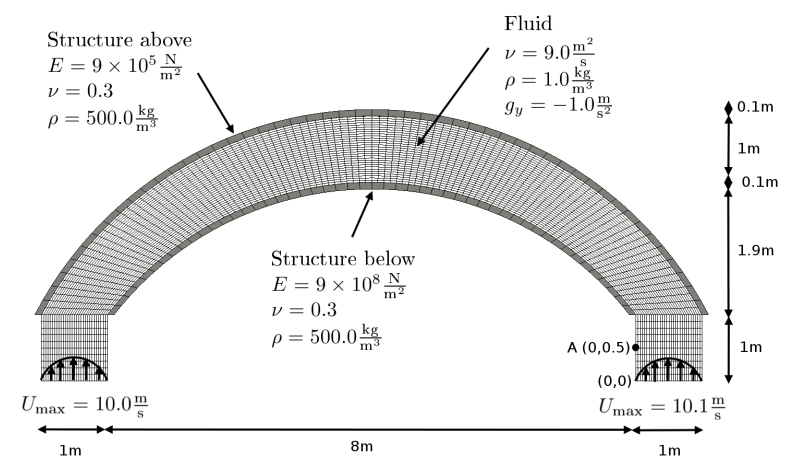

Figure 8: Problem description for the structural instability fully enclosed domain problem. 

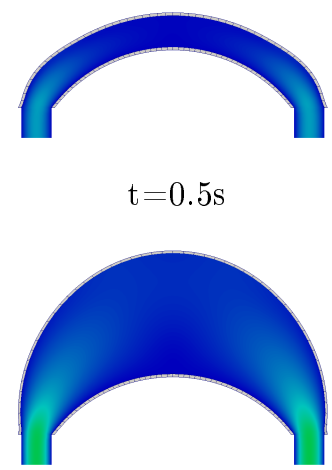

$\mathrm{t}=2.5 \mathrm{~s}$
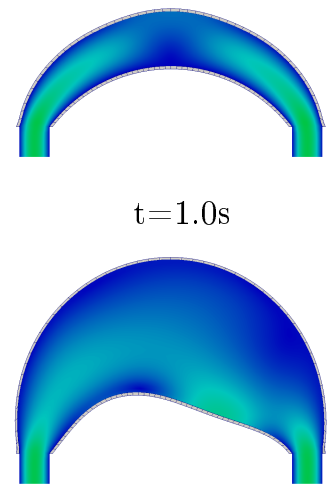

$\mathrm{t}=2.9 \mathrm{~s}$

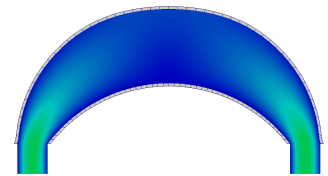

$\mathrm{t}=1.5 \mathrm{~s}$

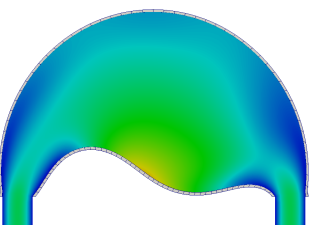

$\mathrm{t}=3.0 \mathrm{~s}$

20

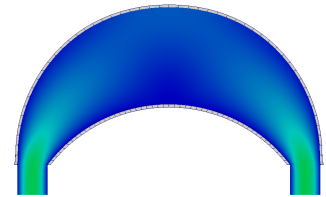

$\mathrm{t}=2.0 \mathrm{~s}$

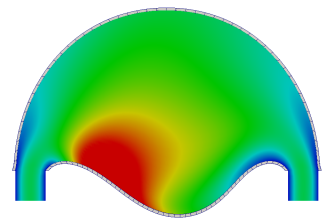

$\mathrm{t}=3.1 \mathrm{~s}$

Figure 9: Velocity fields with structural displacements shown at different time steps for the curved domain fully enclosed test problem.

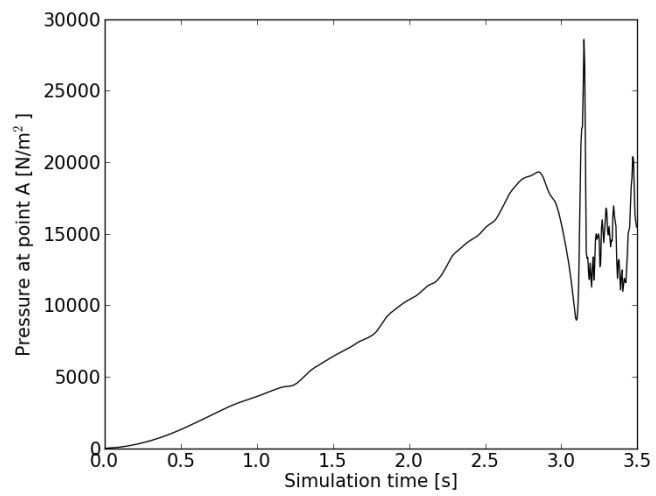

Figure 10: Plot of the pressure level at point A, for the curved domain fully enclosed problem.

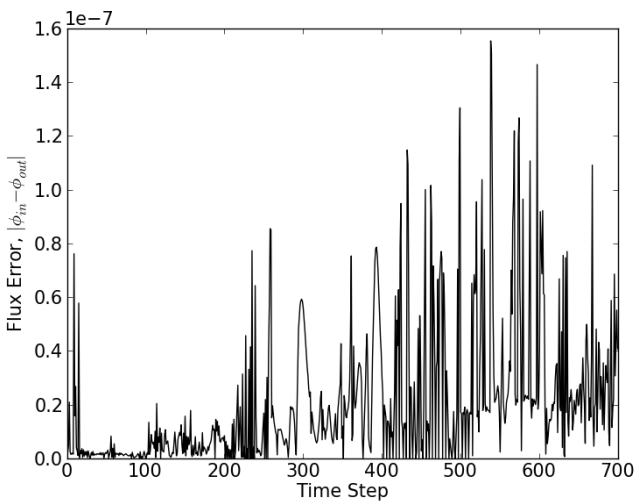

Figure 11: Total flux error made within each of the time steps for the curved domain fully enclosed problem. 

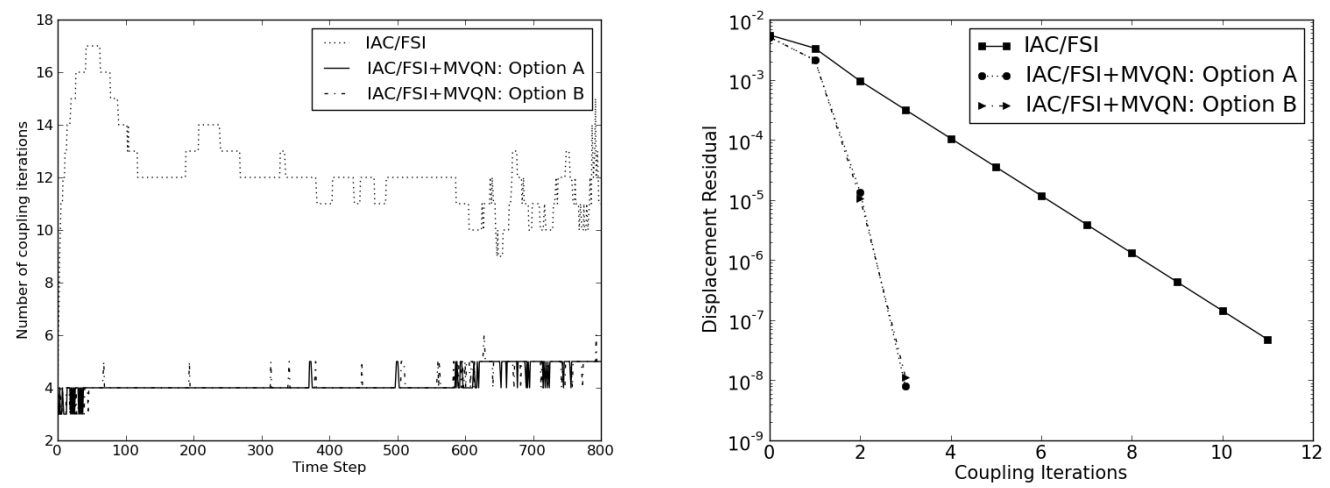

Figure 12: Comparative performance plots for the curved domain, fully enclosed test problem showing the number of coupling iterations within each time step and the typical convergence behavior (shown here for time step 150).

Table 3: Comparison of the number of iterations and relative computational time for the damped structural instability problem.

\begin{tabular}{|c|cccc|}
\cline { 2 - 5 } \multicolumn{1}{c|}{} & Mean & Min & Max & CPU \\
\hline IAC/FSI & 12.21 & 3 & 17 & 2.84 \\
IAC/FSI+MVQN: Option A & 4.21 & 3 & 5 & 1.00 \\
IAC/FSI+MVQN: Option B & 4.23 & 3 & 6 & 1.00 \\
\hline
\end{tabular}

$\mathrm{AC}$ method due the inherent duality in the problem. Firstly, as the structural domain undergoes large deformations, the internal solid stresses increase, resulting in an increased resistance to any further deformation. This is again in contradiction to the increasing inertial forces due to the high structural densities and relatively high velocities (especially once the snap through of the bottom structure occurs). The problem is further complicated by the fluid body force.

In Table 3 we summarize the comparative performance of IAC/FSI and IAC/FSI+MVQN. While IAC/FSI in isolation is entirely capable of solving the problem, the performance is improved through the additional quasiNewton approximations. Figure 12 shows the total number of coupling iterations for each of the time steps as well as the representative convergence behavior (shown here for time step 150). Moreover, as demonstrated previously, the penalty in omitting the additional artificial compressibility displacement $u_{A C}$ is near negligible.

\section{Conclusion}

In this paper we outlined the augmentation of AC/FSI through the inclusion of quasi-Newton methods. We demonstrated how the inclusion of the AC term enabled the solution of fully-enclosed, incompressible, FSI problems. Despite the test load method providing a reasonable means by which to approximate the AC coefficients, the coupling performance of AC/FSI was demonstrated, to at times provide sub-optimal convergence for the test problems investigated in this paper. The additional QN terms in turn provides a means by which to better account for the variety of non-linearities present in the system, and consequently results in an improved convergence behaviour. One of the main attractions of the AC/FSI method is its ability to treat the solid solver and the modified fluid field solver as black-box operators, which is retained for the proposed AC/FSI + QN coupling scheme. The multi-vector update quasi-Newton (MVQN) method was further demonstrated to be a suitable choice for approximating the required system sensitivities. 


\section{Appendix A: IAC/FSI + MVQN Algorithm}

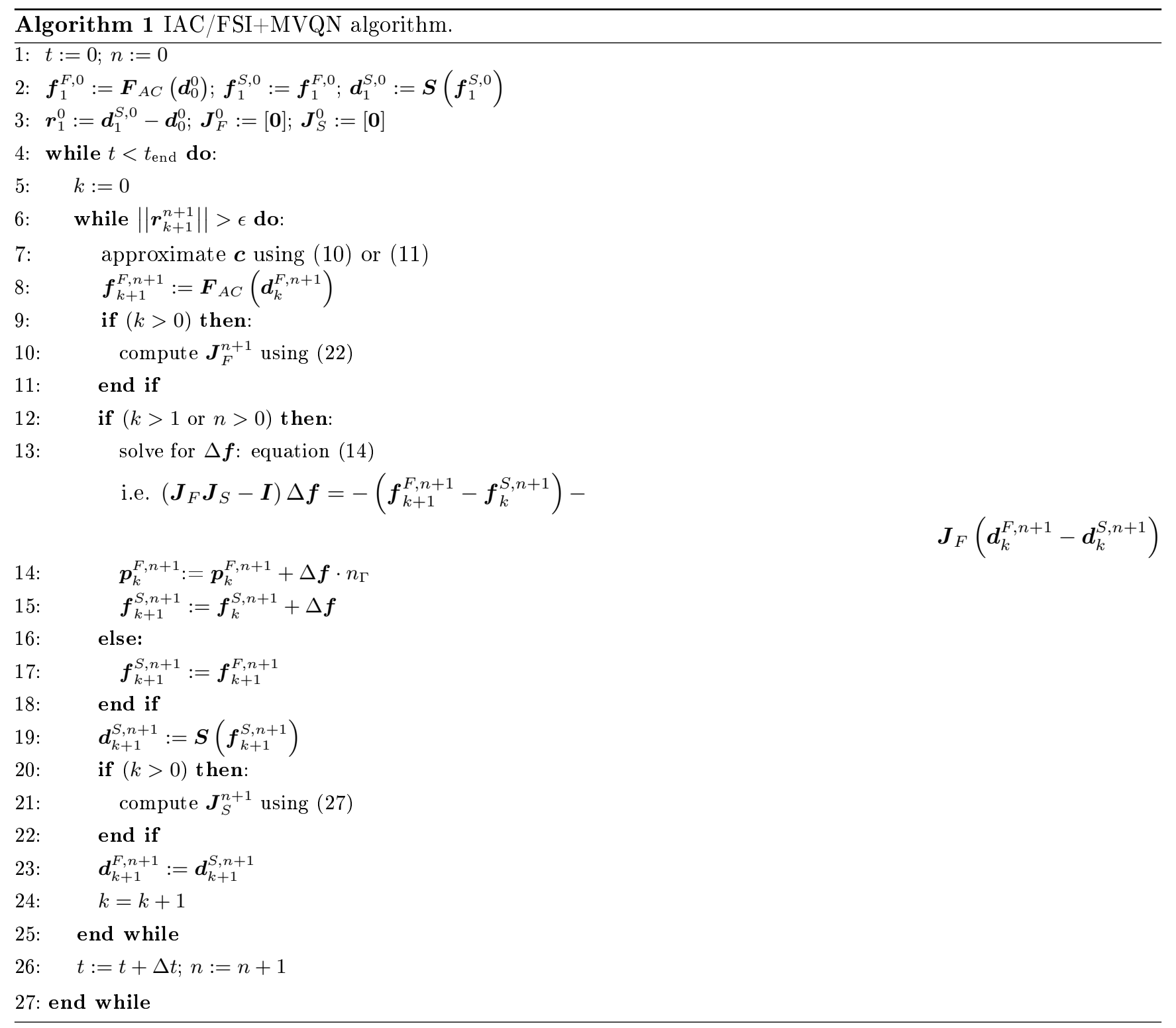

\section{References}

[1] Fluent user's guide, Fluent Inc, Lebanon, 2005.

[2] Openfoam: The open source cfd toolbox user guide, version 2.1.0, 2010.

[3] Santiago Badia, Fabio Nobile, and Christian Vergara. Fluid-structure partitioned procedures based on robin transmission conditions. Journal of Computational Physics, 227(14):7027-7051, 2008.

[4] AEJ Bogaers, S Kok, and T Franz. Strongly coupled partitioned FSI using proper orthogonal decomposition. In Eight South African Conference on Computational and Applied Mechanics, Johannesburg, South Africa, September 2012. 
[5] AEJ Bogaers, S Kok, BD Reddy, and T Franz. Treatment of fully enclosed Fluid Structure Interactions using artificial compressibility. In Third African Congress on Computational Mechanics, AfriCOMP2013, Livingstone, Zambia, July-August 2013.

[6] AEJ Bogaers, S Kok, BD Reddy, and T Franz. Quasi-newton methods for implicit black-box fluid-structure interactions coupling. Comput Meth Appl Mech Eng, 2014, in review.

[7] Alexandre Joel Chorin. A numerical method for solving incompressible viscous flow problems. Journal of computational physics, 2(1):12-26, 1967.

[8] A De Boer, MS Van der Schoot, and H Bijl. Mesh deformation based on radial basis function interpolation. Computers 85 Structures, 85(11):784-795, 2007.

[9] Aukje de Boer, Alexander H van Zuijlen, and Hester Bijl. Comparison of conservative and consistent approaches for the coupling of non-matching meshes. Computer Methods in Applied Mechanics and Engineering, 197(49):4284-4297, 2008.

[10] Joris Degroote. On the similarity between dirichlet-neumann with interface artificial compressibility and robinneumann schemes for the solution of fluid-structure interaction problems. Journal of Computational Physics, 230(17):6399-6403, 2011.

[11] Joris Degroote, Klaus-Jürgen Bathe, and Jan Vierendeels. Performance of a new partitioned procedure versus a monolithic procedure in fluid-structure interaction. Computers $\& 3$ Structures, 87(11):793-801, 2009.

[12] Joris Degroote, Robby Haelterman, Sebastiaan Annerel, Peter Bruggeman, and Jan Vierendeels. Performance of partitioned procedures in fluid-structure interaction. Computers \& structures, 88(7):446-457, 2010.

[13] Joris Degroote, Abigail Swillens, Peter Bruggeman, Robby Haelterman, Patrick Segers, and Jan Vierendeels. Simulation of fluid-structure interaction with the interface artificial compressibility method. International Journal for Numerical Methods in Biomedical Engineering, 26(3-4):276-289, 2010.

[14] Simone Deparis, Marco Discacciati, Gilles Fourestey, and Alfio Quarteroni. Fluid-structure algorithms based on steklov-poincaré operators. Computer Methods in Applied Mechanics and Engineering, 195(41):5797-5812, 2006 .

[15] Wulf G Dettmer and Djordje Perić. On the coupling between fluid flow and mesh motion in the modelling of fluid-structure interaction. Computational Mechanics, 43(1):81-90, 2008.

[16] Guido Dhondt. Calculix crunchix user's manual version 2.5. 2007.

[17] Matthias Heil, Andrew L Hazel, and Jonathan Boyle. Solvers for large-displacement fluid-structure interaction problems: segregated versus monolithic approaches. Computational Mechanics, 43(1):91-101, 2008.

[18] Esko Järvinen, Peter Råback, Mikko Lyly, and Juha-Pekka Salenius. A method for partitioned fluid-structure interaction computation of flow in arteries. Medical engineering 85 physics, 30(7):917-923, 2008.

[19] Christophe Kassiotis, Adnan Ibrahimbegovic, Rainer Niekamp, and Hermann G Matthies. Nonlinear fluidstructure interaction problem. part i: implicit partitioned algorithm, nonlinear stability proof and validation examples. Computational Mechanics, 47(3):305-323, 2011.

[20] U Küttler, M Gee, Ch Foerster, A Comerford, and WA Wall. Coupling strategies for biomedical fluid-structure interaction problems. International Journal for Numerical Methods in Biomedical Engineering, 26(3-4):305$321,2010$.

[21] Ulrich Küttler, Christiane Förster, and Wolfgang A Wall. A solution for the incompressibility dilemma in partitioned fluid-structure interaction with pure dirichlet fluid domains. Computational Mechanics, 38(45):417-429, 2006. 
[22] Hermann G Matthies and Jan Steindorf. Partitioned strong coupling algorithms for fluid-structure interaction. Computers \& Structures, 81(8):805-812, 2003.

[23] Peter Raback, Juha Ruokolainen, Mikko Lyly, and Esko Järvinen. Fluid-structure interaction boundary conditions by artificial compressibility. In ECCOMAS CFD, volume 2001, 2001.

[24] Kristiaan Riemslagh, Jan Vierendeels, and Erik Dick. An efficient coupling procedure for flexible wall fluidstructure interaction. 2000.

[25] Stefan Turek and Jaroslav Hron. Proposal for numerical benchmarking of fluid-structure interaction between an elastic object and laminar incompressible flow. Fluid-Structure Interaction: Modelling, Simulation, Optimisation, 53:371, 2007.

[26] Jan Vierendeels, Lieve Lanoye, Joris Degroote, and Pascal Verdonck. Implicit coupling of partitioned fluidstructure interaction problems with reduced order models. Computers \&3 structures, 85(11):970-976, 2007. 